\title{
Role of Ultrasound and Tru-Cut Biopsy in Breast Lumps: A Contemporary Review
}

\author{
Nagam Amarnath ${ }^{1} \odot$, Vinoth Kumar ${ }^{2}, \mathrm{CP}$ Ganesh Babu ${ }^{3}$
}

\begin{abstract}
Background: Ultrasonography (USG) is one of the most commonly used and important imaging modalities in assessing breast lumps. It helps majorly in differentiating cystic and solid lesions. Tru-Cut biopsy (TCB) is less invasive and can be done easily with minimal deformity and scarring. The two main objectives of percutaneous biopsy techniques are to achieve a high degree of accuracy and to give information about the tumor (type, grade, invasion, hormonal receptors, HER-2 NEU, etc.). This review article is an attempt to evaluate the superiority of one test over the other based on the available literature.

Methods: Primary relevant publications obtained from PubMed and Google Scholar were included. Duplications of articles from various databases were eliminated. The articles were reviewed based on the role of ultrasound and TCB in the evaluation of breast lumps.

Results: Both USG and TCB have similar diagnostic accuracy in the evaluation of breast lumps and superiority could not be established.

Keywords: Benign breast diseases, Breast cancer, Core needle biopsy, Histopathology, Tru-Cut biopsy, Ultrasound.

Annals of SBV (2021): 10.5005/jp-journals-10085-9110
\end{abstract}

\section{INTRODUCTION}

In women with palpable breast lumps, the most common worry about breast cancer is what brings them to the clinician. Carcinoma of the breast is one of the leading causes of death due to cancer in women. Early detection of these conditions is needed and lack of detection at an early stage is responsible for presentation at late stages when it cannot be cured completely. ${ }^{1}$ Umanah et al. in a study on the adolescent population in Africa found out that the most common breast masses are fibroadenoma and fibrocystic changes. Early detection also helps in reducing mortalities. Optimal management of palpable breast lumps includes triple assessment that includes a physical examination, mammography, and percutaneous biopsy. ${ }^{2}$

Ultrasound is one of the most commonly used and important imaging modalities in assessing breast lumps. It helps majorly in differentiating cystic and solid lesions. Studies have shown that ultrasound has high sensitivity and specificity for the detection of invasive breast carcinoma. Wild and Neal in 1952 were the first to report the use of diagnostic sonography in the evaluation of breast diseases. $^{3}$

Tru-Cut biopsy is less invasive and can be done easily and with minimal deformity and scarring. Complications include hematoma and infection. It also costs less. ${ }^{4,5}$ The two main objectives of percutaneous biopsy techniques are to achieve a high degree of accuracy and to give information about the tumor (e.g. type, grade, invasion, hormonal receptors, HER-2 neu, etc.). Preoperative core needle biopsy is becoming the standard of care in breast carcinoma. Core needle biopsy can be performed using automated biopsy guns especially for the breast. Parker et al. reported real-time ultrasound monitoring during needle biopsy in $1983 .{ }^{5-8}$ Fine needle aspiration cytology (FNAC) lacks important information that TCB provides, and this information is important for the formulation of a preoperative plan by the surgeon and oncologist. Tru-Cut biopsy provides all the reliable information and guides the surgeon and oncologist. ${ }^{9,10}$

There are studies with conflicting results trying to establish the superiority of one over the other. In this review article, we

\footnotetext{
${ }^{1-3}$ Department of General Surgery, Mahatma Gandhi Medical College and Research Institute, Puducherry, India

Corresponding Author: Vinoth Kumar, Department of General Surgery, Mahatma Gandhi Medical College and Research Institute, Puducherry, India, Phone: +918110011955, e-mail: vino8683@gmail. com

How to cite this article: Amarnath N, Kumar V, Babu CPG. Role of Ultrasound and Tru-Cut Biopsy in Breast Lumps: A Contemporary Review. Ann SBV 2021;10(2):42-46.

Source of support: Nil

Conflict of interest: None
}

evaluate the performance of ultrasound and TCB in the diagnosis of breast lumps.

\section{Materials and Methods}

Relevant publications collected from PubMed and Google Scholar were included in the study. The key search words used were "ultrasound" and "Tru-Cut biopsy." No restriction based on date of publication was enforced. Duplications of publications from multiple databases were eliminated. A brief contemporary overview of ultrasound and TCB was prepared.

\section{Review of Literature Embryology}

In the fifth or sixth week of fetal growth, two grouped ectoderms (papillae, milk lines) of the stomach wall surface can be seen in the embryo. When the ectoderm grows internally to form the primary tissue buds in the stroma, each breast will form. Thereafter, the main buds start the growth of 15-20 additional buds. The main (mastoid) duct develops, which causes a superficial breast pit. In the early stage, mesenchymal hyperplasia turns the mammary glands into nipples. If the pit cannot be increased over the skin level, it will 
trigger upside down nipple areas. This congenital malformation occurs in $4 \%$ of infants. Breast enlargement may be evident and also may produce discharge, called witch milk. These transient occasions happen in feedback to mother's hormonal agents that cross the placenta. The breast does not establish in women until puberty. When it expands in action to ovarian estrogen and progesterone, this causes the proliferation of epithelial as well as connective tissue elements.

\section{Functional Composition}

Breasts are modified sweat glands composed of wattles, and lobules spread in adipose tissue as well as connective tissue. The air duct drains from each lobule. They integrate to develop a breast air duct, which drains from each lobe. The mammary ducts combine simply below the nipple to form the mammary sinuses. The practical secretory device in lactation is the terminal lobular unit.

The breast is made up of 15-20 lobes. Cooper's suspensory ligament from pectoral fascia inserts into the dermis. Retromammary space is the space between the pectoral fascia and breast parenchyma. Axillary tail of Spence extends from the upper outer quadrant. The upper outer quadrant has more breast tissue. The size, contour, and also thickness of breasts vary substantially between individuals. Prenatal breasts are hemispherical, and the top of the nipple area is undoubtedly level. With hormone stimulation during pregnancy and lactation, the breasts end up being bigger and their volume and thickness also increases. When aging, the breasts become level, loosened, and also droop, besides reducing in volume.

\section{Blood Supply, Innervation, and Lymphatics}

The primary blood supply to the breast comes from (1) the perforated branch of the interior mammary artery; and (2) the side branch of the posterior intercostal artery, as well as the branch from the axillary artery, consisting of the greatest thoracic, lateral, and thoracic branches of the thoracic artery. The second, third, and fourth former intercostal perforators as well as branches of the internal mammary artery create the median mammary artery in the breast. The veins of the breast and also chest wall surface adhere to the direction of the artery, and the veins flow to the armpit.

The external skin branches of the third to sixth intercostal nerves provide sensory nerves for the breast as well as the anterolateral upper body wall surface. Lymphatic vessels are usually alongside capillaries. The boundary of the axillary lymphatic drainage is not well specified. The axillary lymph node groups recognized by the surgeon are: (1) the axillary vein group (outer side), consisting of four to six lymph nodes, located in the middle or back of the vein and receiving most of the upper limb lymphatic drainage; (2) external breast glands group (anterior or pleural group), consisting of five or six lymph nodes, which are connected to the lateral thoracic vessels along the lower border of the pectoralis minor muscle and receiving most of the lymphatic drainage from the outside of the breast; (3) the central group consisting of three or four groups of lymph nodes. These lymph nodes are buried in the axillary fat, located at the back of the small axillary muscles, and receive lymph drainage from the axillary veins, outside the mammary glands and the shoulder scapular group directly from the breast; (4) The subclavian group consists of 6-12 groups of lymph nodes, which are located behind the upper border of the pectoralis minor muscle and drain from all other axillary lymph node groups; and (5) the interpectoral muscles (Rotter's node), composed of one to four lymph nodes, are located between the pectoralis major and pectoralis minor muscles and receive lymphatic drainage directly from the breast. The lymph fluid passing through the interpleural lymph node cluster directly enters the center and subclavian cluster.

\section{Classification of Breast Lumps}

The breast is composed of unique epithelium and stroma, which might cause benign and fatal lesions. Visible discrete masses are additionally common and are identified from regular blemishes (or "lumps") in the breast. The most usual palpable lesions are invasive cancer, fibroadenoma, and cysts. When the dimension of the breast mass reaches at least $2 \mathrm{~cm}$, the breast mass usually becomes evident. Palpable lumps are most typical in premenopausal ladies, but with age, the possibility of apparent lumps ending up being malignant increases. As an example, in ladies under 40 , just $10 \%$ of breast swellings are fatal whereas in women over 50 , about $60 \%$ of swellings are fatal. About $50 \%$ of cancer cells occur in the top outer quadrant, $10 \%$ in each of the remaining quadrants, and $20 \%$ in the middle or subareolar location.

\section{Diagnosis of Breast Lumps}

Breast abnormalities should always be examined by three-way assessment: professional examination, imaging evaluation (mammography and also ultrasonogram), and histopathology.

\section{Ultrasonography}

Currently, ultrasonography (USG) is an adjunctive diagnostic tool indicated for evaluation of specific abnormalities detected by either clinical examination or by mammography or for guiding percutaneous biopsy and localization. In the imaging of young women under 30 years, ultrasonography is used as the first imaging study in the evaluation of a palpable mass. With the introduction of high-frequency $(>7 \mathrm{mHz}$ ) transducers, the differentiation between solid and cystic masses has become more reliable than was the case with older equipment and the characterization of solid masses as benign or suggestive of malignancy can now be established with confidence. Recent studies have demonstrated the ability of breast ultrasonography to detect small, mammographically occult breast carcinomas in women with dense breast tissue. A cancer detection rate of $0.3-0.46 \%$ has been reported, similar to that of mammographic screening (Tables 1 and 2).

\section{Imaging-guided Breast Biopsy}

Traditionally, open surgical breast biopsy has been the gold standard in diagnosing breast lesions; nonpalpable lesions undergo open biopsy after ultrasonography or mammography guided wire localization. The costs and relative morbidity associated with the surgery in conjunction with detection of large number of non-palpable breast lesions on screening programs have lead to the development of percutaneous imaging guided breast biopsy methods to replace surgical biopsies. Imaging guided breast biopsy is most often performed under ultrasonographic or stereotactic guidance and for specific indications, under MRI guidance. Complications are rare, $<2 \%$ for core biopsies and $4 \%$ for vacuum assisted biopsies; the complications include bleeding, hematomas, vasovagal reactions and infections. Early work with percutaneous breast biopsy involved FNA which has in most centers been replaced by cutting needles (automated large core needles) and recently by directional vacuum assisted devices. ${ }^{11}$ 
Role of Ultrasound and Tru-Cut Biopsy in Breast Lumps

Table 1: Literature review matrix of role of ultrasound comparing similar articles and their results

\begin{tabular}{|c|c|c|c|c|c|c|c|}
\hline S. No. & Indices of screening test & Singh et al. ${ }^{19}$ & Lister et al. ${ }^{20}$ & Gonzaga et al. ${ }^{21}$ & Smallwood et al. ${ }^{22}$ & Sood et al. ${ }^{23}$ & Lehman et al. $^{24}$ \\
\hline 1. & Sensitivity (\%) & 95 & 93 & 92.5 & 82 & 80.1 & 95.7 \\
\hline 2. & Specificity (\%) & 94.7 & 97 & 62.8 & 89 & 88.4 & 89.2 \\
\hline 3. & Positive predictive value (\%) & 91.2 & 68 & 68.1 & - & - & 13.2 \\
\hline 4. & Negative predictive value (\%) & - & 99 & 99.5 & - & - & 99.9 \\
\hline 5. & Accuracy (\%) & 92 & 97 & - & - & - & - \\
\hline
\end{tabular}

Table 2: Literature review matrix of role of TCB comparing similar articles and their results

\begin{tabular}{llcccccc}
\hline S. No. & Indices of screening test & Bdour et al. $^{25}$ & Rikabi et al. $^{26}$ & Faizi et al. $^{27}$ & Oluwasola et al. $^{28}$ & Dimitrov et al. $^{29}$ & Caruso et al. $^{30}$ \\
\hline 1. & Sensitivity (\%) & 97 & 95.1 & 96.7 & 86 & 98.6 & 92 \\
2. & Specificity (\%) & 100 & 100 & 70 & 71 & 100 & 100 \\
3. & Positive predictive value (\%) & - & 100 & 90.6 & - & 100 & 100 \\
4. & Negative predictive value (\%) & - & 97.2 & 87.5 & - & 80 & - \\
5. & Accuracy (\%) & - & 98.2 & 90 & 80.4 & 98.73 & 86 \\
\hline
\end{tabular}

\section{Ultrasonographic Guidance}

Ultrasonographic guidance may be regarded as the first-line method for percutaneous biopsy of lesions visible by ultrasonography in terms of real-time visualization of the needle, patient comfort, lack of ionizing radiation, and low cost. With the help of ultrasonography, access to all areas of breast and axilla, even in small breasts can be reached. With ultrasonography-guided 14-gauge biopsies, sensitivities and specificities of $100 \%$ have been reported. However, microcalcifications and a small subset of masses cannot be reliably detected by ultrasonography. The ultrasonography-guided biopsies of tiny lesions and deep localizations may be complicated and time-consuming, and stereotactic guidance in these situations may be preferable. ${ }^{11}$

\section{Core Needle Biopsy}

Overall, it was observed that TCB was better than FNAC in the medical diagnosis of both malignant as well as benign lesions although the result was not statistically considerable. Tru-Cut biopsy additionally fell short to identify instances of lymphoma and also fibrosarcomas in maintaining with the experience of Elesha et al. at Lagos University Teaching Hospital. This may be a restriction caused by the small size of the tissue obtained by core-needle biopsy (CNB). ${ }^{12}$

Benefits of TCB include stipulation of appropriate product for histology when compared to FNAC. It is likewise less expensive than open biopsy and also avoids the resulting scar. It can be performed in the office or other locations. Its tattooing impact assists in succeeding recognition of needle tract at the time of clear-cut resection. In contrast to FNAC, it causes extra pain and has a greater possibility for causing discoloration, hematoma, and infection. It is additionally tough to execute in lesions that are less than $1 \mathrm{~cm} .{ }^{13}$ Logan-Young et al. observed that sensible use of FNAC as well as CNB in theory ought to lead to better accuracy in medical diagnosis of breast cancer cells, as well as fewer unnecessary open surgical biopsies, hence lowering the costs. ${ }^{14}$

A research study assessed 143 clients with palpable breast masses who were reviewed via professional examination, mammography, ultrasound, magnetic resonance imaging (MRI), and FNAC, and it was concluded that a combination of cytology and ultrasound can properly diagnose breast diseases. ${ }^{15}$ In an 8-year research study, Bassett et al. examined the effectiveness of $\mathrm{X}$-ray and also ultrasound for women under 35 years (1,016 women).
This study revealed that mammography is not extremely valuable for women younger than 35 years. However, ultrasonography avoids unnecessary breast lump biopsy. As a result, USG has been shown to be a dependable research study for women under 35 . Yet this is not appropriate for spotting unattainable breast lumps, so it is likely to miss out on unattainable early breast malignancies. ${ }^{16}$

In 1996, Vetto JJ et al. proposed the "customized triple examination" (C/E, USG, FNAC/CNB) to study women under the age of 55 , who were evaluated for mammograms. The study showed high specificity for malignant growths and had a negative anticipation of $100 \%$. They concluded that using modified triple assessment (MTT) to diagnose palpable bust masses in young women has a high diagnostic accuracy. ${ }^{17}$

A study by Jayakar et al. (2013) reviewed the efficiency of ultrasound instead of mammography in the assessment of young women with evident breast masses in a regular "three-way trial." They ended saying that breast masses detected as deadly can be completely treated. ${ }^{18}$

\section{Comparison of Ultrasound and TCB}

In 2008, Singh et al. in their study on ultrasonographic evaluation of 100 cases of palpable breast masses done from 2003 to 2005 said that the diagnosis on ultrasound was made considering four features, namely, shape, margins, width, A:P ratio, and echogenicity. Diagnosis was confirmed by FNAC or histopathology. They concluded saying that its sensitivity for the detection of cystic lesions is high and it can definitely differentiate cystic from solid lesions. Role of ultrasound needs further evaluation before it can be used as a screening tool in diagnosis of breast carcinoma. ${ }^{19}$

In 1998, Lister et al. performed a prospective observational analytical study on the accuracy of ultrasound in detecting invasive malignancy in clinically benign, discrete, symptomatic breast lumps prior to mammography on 205 clinically benign breast masses where ultrasound findings were compared with FNAC, CNB, and surgical findings. They found that ultrasound is better than mammogram and is a useful adjunct to FNA/CNB in evaluating clinically symptomatic benign breast lesions. ${ }^{20}$

In 2010, Gonzaga et al. did a study on the accuracy of ultrasound in diagnosis of palpable breast masses in 80 cases with palpable breast masses in the radiology department in Mulago Hospital. Impression derived from ultrasound was correlated with histopathological findings. They concluded that ultrasound 
significantly differentiates solid and cystic lumps and it should be used for investigating breast masses which are palpable. ${ }^{21}$

In 1986, Smallwood et al. in their retrospective study on 1,000 patients with symptomatic breast disease who underwent USG and mammogram in Southampton compared the accuracy of ultrasound with that of mammogram. They concluded that ultrasound is more sensitive and specific than mammogram and that USG is an important adjunct to mammogram for the evaluation of breast lesions preoperatively. ${ }^{22}$

In 2018, Sood et al. in their systematic review and meta-analysis on 26 articles from 2000 to 2018 concluded that ultrasound is an effective primary tool in evaluation of breast cancers and can be of utmost benefit in places where mammogram is unavailable. ${ }^{23}$

In 2012, Lehman et al. studied the accuracy and value of breast ultrasound for primary imaging evaluation of symptomatic women between 30 and 39 years. They reviewed all electronic records of breast ultrasound and mammogram done between 2002

and 2006 and compared them with final clinical records. Conclusion was that ultrasound should be the primary modality for imaging and mammogram as an adjunct is of low value. ${ }^{24}$

In 2008, Bdour et al. in their prospective study from 2006 to 2007 on 175 patients with breast lumps where results of FNAC and CNB in the diagnosis of breast cancer were compared said that CNB is more accurate than FNAC and is useful in suspicious breast lump evaluation. CNB also helps for neoadjuvant therapy in cases indicated. ${ }^{25}$

In 2013, Rikabi et al. conducted a study on the usefulness of TCB in the diagnosis of breast lesions. It is an observational noninterventional cross-sectional review that covered all TCB reports of a mass or lesion observed in patients admitted between 2008 and 2010. A total of 275 TCBs were performed during the study period. Their conclusion was that TCB has high accuracy and can be used as an alternative for FNAC in evaluating breast lesions. ${ }^{26}$

In 2012, Faizi conducted a study to determine the diagnostic accuracy of TCB in the detection of breast lumps, taking postsurgical histopathology as the gold standard. It was a crosssectional survey, from February 7, 2011, to February 6, 2012 and a total of 40 married and unmarried women over 15 years of age with clinically palpable lump in the breast were recruited in this study. All female patients having acute breast abscess, previously operated and nonmotivated patients were excluded from study. They concluded that unnecessary surgery can be avoided with the use of TCB and TCB could be helpful in evaluation of suspicious breast lesions. ${ }^{27}$

In 2015, Oluwasola et al. in their retrospective study on patients with TCB and follow-up excisional biopsy or mastectomy for breast lumps in 51 patients at the University College Hospital, Ibadan, concluded that TCB is comparable to excisional biopsy and can be most accurate when used with image guidance. ${ }^{28}$

In 2016, Dimitrov et al. conducted a retrospective clinical study on the diagnostic value of TCB in diagnosing breast lesions in 79 patients between 2014 and 2015 and concluded that TCB is a safer, cheaper, and easier alternative to FNAC in evaluating and diagnosing lesions of breast. ${ }^{29}$

In 1998, Caruso et al. studied core biopsy as an alternative to FNAC in diagnosis of breast tumors. This was a 5-year study on 80 cases to evaluate the diagnostic usefulness of CNB and to reduce intraoperative biopsies. They concluded that CNB is a useful and definitive method in the evaluation of breast lumps. ${ }^{30}$

\section{Conclusion}

Both USG and TCB have similar diagnostic accuracy in breast lumps. The choice of investigation depends on the investigators and the physician. Superiority of one over the other cannot be achieved based on the available literature.

\section{ORCID}

Nagam Amarnath $\odot$ https://orcid.org/0000-0001-6905-9180

\section{References}

1. Umanah IN, Akhiwu W, Ojo OS. Breast tumours of adolescents in an African population. Afr J Paediatr Surg 2010;7(2):78-80. DOI: 10.4103/0189-6725.62849.

2. Tabár L, Péntek Z, Dean PB. The diagnostic and therapeutic value of breast cyst puncture and pneumocystography. Radiology 1981;141(3):659-663. DOI: 10.1148/radiology.141.3.7302219.

3. Wild JJ, Reid JM. Further pilot echographic studies on the histologic structure of tumors of the living intact human breast. Am J Pathol 1952;28(5):839-861.

4. Percutaneous imaging-guided core breast biopsy. Am J Roentgenol 174:5 Available from: https://www.ajronline.org/doi/full/10.2214/ ajr.174.5.1741191.

5. US-guided 14-gauge core-needle breast biopsy: results of a validation study in 1352 cases. Radiology. Available from: https://pubs.rsna.org/ doi/10.1148/radiol.2482071994.

6. Vitums VC. Percutaneous needle biopsy of the lung with a new disposable needle. CHEST 1972;62(6):717-719. DOI: 10.1378/ chest.62.6.717.

7. US-guided automated large-core breast biopsy. Radiology. Available from: https://pubs.rsna.org/doi/abs/10.1148/radiology.187.2. 8475299.

8. Donald JJ, Coral A, Shorvon PJ, Lees WR. Ultrasound guided core biopsy in AIDS: experience in six patients. Br Med J Clin Res Ed 1988;296(6622):606-607. DOI: 10.1136/bmj.296.6622.606.

9. Pinder SE, Elston CW, Ellis IO. The role of pre-operative diagnosis in breast cancer. Histopathology 1996;28(6):563-566. DOI: 10.1046/ j.1365-2559.1996.d01-465.x.

10. Probabilistic approach to the reporting of fine-needle aspiration cytology of the breast. Abstract-Europe PMC Available from: http:// europepmc.org/article/MED/9100534.

11. Sonographically guided core biopsy of the breast: comparison of 14-gauge automated gun and 11-gauge directional vacuumassisted biopsy methods. Available from: https://www.research gate.net/publication/237308879_Sonographically_Guided_Core_ Biopsy_of_the_Breast_Comparison_of_14Gauge_Automated_ Gun_and_11Gauge_Directional_Vacuum-Assisted_Biopsy_ Methods.

12. Primary malignant lymphoma of the breast. Available from: http:// erepository.uonbi.ac.ke/handle/11295/10269.

13. Scopa CD, Koukouras D, Spiliotis J, Harkoftakis J, Koureleas S, Kyriakopoulou D, et al. Comparison of fine needle aspiration and tru-cut biopsy of palpable mammary lesions. Cancer Detect Prev 1996;20(6):620-624.

14. Logan-Young W, Dawson AE, Wilbur DC, Avila EE, Tomkiewicz ZM, Sheils LA, et al. The cost-effectiveness of fine-needle aspiration cytology and 14-gauge core needle biopsy compared with open surgical biopsy in the diagnosis of breast carcinoma. Cancer 1998;82(10):1867-1873. DOI: 10.1002/(sici)1097-0142(19980515) 82:10<1867::aid-cncr8>3.0.co;2-k.

15. Hardy JR, Powles TJ, Judson I, Heron C, Williams M, Cherryman G, et al. How many tests are required in the diagnosis of palpable breast abnormalities? Clin Oncol R Coll Radiol G B 1990;2(3):148-152. DOI: 10.1016/s0936-6555(05)80148-7. 
16. Bassett LW, Ysrael M, Gold RH, Ysrael C. Usefulness of mammography and sonography in women less than 35 years of age. Radiology 1991;180(3):831-835. DOI: 10.1148/radiology.180.3.1871302.

17. Vetto JT, Pommier RF, Schmidt WA, Eppich H, Alexander PW. Diagnosis of palpable breast lesions in younger women by the modified triple test is accurate and cost-effective. Arch Surg 1996;131(9):967-974. DOI: 10.1001/archsurg.1996.014302 10065012.

18. IJAR. Available from: https://www.worldwidejournals.com/indianjournal-of-applied-research-(IJAR)/recent_issues_pdf/2016/April/ April_2016_1461302804_02.pdf.

19. Singh K, Azad T, Gupta GD. The accuracy of ultrasound in diagnosis of palpable breast lumps. Clin Radiol 2008;10(4):186-188. DOI: 10.1016/ S0009-9260(98)80167-0.

20. Lister D, Evans AJ, Burrell HC, Blamey RW, Wilson ARM, Pinder $\mathrm{SE}$, et al. The accuracy of breast ultrasound in the evaluation of clinically benign discrete, symptomatic breast lumps. Clin Radiol 1998;53(7):490-492. DOI: 10.1016/S0009-9260(98)80167-0

21. Gonzaga MA. How accurate is ultrasound in evaluating palpable breast masses? Pan Afr Med J. 2010;7:1. Available from: https://www. ncbi.nlm.nih.gov/pmc/articles/PMC3172638/.

22. Smallwood JA, Guyer P, Dewbury K, Mengatti S, Herbert A, Royle GT, et al. The accuracy of ultrasound in the diagnosis of breast disease. Ann R Coll Surg Engl 1986;68(1):19-22. DOI: 10.1200/ JGO.19.00127.

23. Sood R, Rositch AF, Shakoor D, Ambinder E, Pool K-L, Pollack E, et al. Ultrasound for breast cancer detection globally: a systematic review and meta-analysis. J Glob Oncol 2019;(5):1-17. DOI: 10.1200/ JGO.19.00127.

24. Lehman $\mathrm{CD}$, Lee $\mathrm{Cl}$, Loving VA, Portillo MS, Peacock S, DeMartini WB. Accuracy and value of breast ultrasound for primary imaging evaluation of symptomatic women 30-39 years of age. Am J Roentgenol 2012;199(5):1169-1177. DOI: 10.2214/AJR.12.8842.

25. Bdour M, Hourani S, Mefleh W, Shabatat A, Karadsheh S, Nawaiseh $\mathrm{O}$, et al. Comparison between fine needle aspiration cytology and tru-cut biopsy in the diagnosis of breast cancer. J Surg Pak 2008; 13:19-21.

26. Rikabi A, Hussain S. Diagnostic usefulness of tru-cut biopsy in the diagnosis of breast lesions. Oman Med J 2013;28(2):125-127. DOI: 10.5001/omj.2013.32.

27. Faizi K, Hameed T, Azim K, Mukhtar S. Diagnostic accuracy of trucut biopsy in the detection of breast lumps. Pak J Med Health Sci 2012;6:979-981. DOI: 10.1515/ jbcr-2016-0018.

28. Diagnostic accuracy of tru-cut biopsy of breast lumps at University College Hospital, Ibadan. Available from: https://europepmc.org/ article/med/26937529.

29. Dimitrov DD, Karamanliev MP, Deliyski TS, Gabarski AV, Vatov PP, Gencheva RO, et al. Diagnostic value of tru-cut biopsy in diagnosing breast lesions. J Biomed Clin Res 2016;9(2):126-129. DOI: 10.1515/jbcr2016-0018.

30. Caruso ML, Gabrieli G, Marzullo M, Pirrelli M, Rizzia E, Sorino F. Core biopsy as alternative to fine-needle aspiration biopsy in diagnosis of breast tumors. Oncologist 1998;3(1):45-49. DOI: 10.1634/ theoncologist.3-1-45. 